\title{
Accurate Temperature Drift model of MOSFETs Mobility for Analog Circuits
}

\author{
Kazufumi Watanabe, Tatsufumi Hamada, Koji Kotani, Akinobu Teramoto*, \\ Shigetoshi Sugawa, and Tadahiro Ohmi* \\ Graduate School of Engineering, Tohoku University, \\ *New Industry Creation Hatchery Center, Tohoku University. \\ Aza-Aoba 10, Aramaki, Aoba-ku, Sendai 980-8579, Japan, \\ (Phone: +81-22-217-3977 / Fax: +81-22-217-3986). \\ Email:k-watanabe@fff.niche.tohoku.ac.jp
}

\begin{abstract}
An accurate compact MOSFETs model that can deal with effects of temperature drift is prerequisite for analog circuit design. Although the mobility behavior at high vertical field region is important for simulating analog circuits, however current physics based models neglect the temperature dependence of that at this region. In this paper, a new mobility model at high vertical field region is proposed. The developed model can capture the mobility behavior for different surface orientations, since this model is based on the essential physics. The model accuracy is verified by the experimental data.
\end{abstract}

\section{Introduction}

Aggressive developments of integration technologies make it possible to integrate many MOSFETs and devices on one chip. To realize many functions on a chip, these integration technologies are aggressively applied. Consequently, the complex system that had been configured by many chips in the past is realized on a single chip [1][2]. This chip is, in general, called as "System on a Chip (SoC)". The components of SoC can roughly be divided into two parts, which are digital and analog circuit parts. The analog circuit parts have the sensitive characteristics to noise, environment temperature and so on. Thus, the accurate MOSFET model that can predict these characteristics at any situation is required to design a SoC chip. Especially, the temperature drift of MOSFETs characteristics due to chip heating is one of the serious problems in designing SoC. However, the accurate model that can predict temperature drift had not been reported and implemented in SPICE model.

In addition, the MOSFETs fabricated on $\mathrm{Si}(110)$ surface attract much attention due to the possibility of realizing a balanced CMOS using high mobility of pMOSFETs and low Flicker noise [3]. The model should have the adaptability for the MOSFETs fabricated on (110) surface.

In this paper, we have developed the temperature drift model of MOSFETs based on the physics. To analyze the carrier scattering, we applied a Monte-Carlo simulator in many particle system.

The accuracy of the developed model is verified by the experimental data. The description of the developed model is compact as well as can capture the MOSFET behavior at any temperature although surface orientation had changed. 


\section{Simulation Methods}

The mobility behavior of MOSFETs is the hart of describing I-V characteristics. The mobility can be classified into three parts, which are the Coulomb, Phonon and Surface-Roughness scattering [4-6]. Especially, the mobility at the high vertical field region (high $\mathrm{E}_{\text {eff }}$ ) is very important to design analog circuits. In this paper, we focus on the modeling of surface-roughness (SR) scattering since the SR scattering is dominant in high $\mathrm{E}_{\text {eff }}$ region. The following equation shows a model equation of general SR mobility.

$$
\mu_{s r}=\frac{\mu_{s r 0}}{E_{e f f}^{2.0}}
$$

A SR scattering does not have temperature dependency in conventional model, meanwhile the temperature dependency has been observed recently [6]. We applied a Monte-Carlo simulator to clarify the scattering mechanism at high $\mathrm{E}_{\text {eff }}$ region. The scattering processes are summarized in Table-I.

Table-I. Details of phonon scatterings. This condition is used to simulate the scattering processes in Monte-Carlo simulator.

\begin{tabular}{|c|c|c|c|c|}
\hline Model & Process & $\begin{array}{c}\text { Phonon Energy } \\
{[\mathrm{meV}]}\end{array}$ & $\begin{array}{c}\text { Deformation } \\
\text { Potential } \\
{\left[\times 10^{8} \mathrm{eV} / \mathrm{cm}\right]}\end{array}$ & Mode \\
\hline \multirow{7}{*}{} & f-proc. & $19.0(220.6 \mathrm{~K})$ & 0.3 & TA-Phonon \\
& f-proc. & $47.5(551.4 \mathrm{~K})$ & 2.0 & LA-Phonon \\
& f-proc. & $59.1(686.1 \mathrm{~K})$ & 2.0 & TO-Phonon \\
\cline { 2 - 5 } & g-proc. & $12.1(140.5 \mathrm{~K})$ & 0.5 & TA-Phonon \\
& g-proc. & $18.6(215.9 \mathrm{~K})$ & 0.8 & LA-Phonon \\
& g-proc. & $62.2(722.1 \mathrm{~K})$ & 19.2 & LO-Phonon \\
\hline
\end{tabular}

\section{Simulation Results and Modeling of Scattering Process}

Figure 1 shows the simulation result about valley-I \& II, and valley-III occupations of electrons at certain temperature.

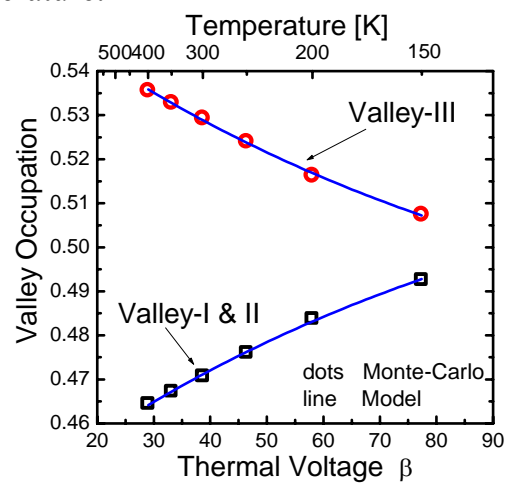

Figure 1 The calculated and simulated results at a given temperature. The developed model can capture the simulation results. 
Where, the f-process through LA- and TO modes, and g-process through LO-mode are dominant from the simulaton result. we modeled this scattering process.

$$
\begin{aligned}
P_{I I I}= & P_{I I I}^{0}+\bar{a}_{\text {del }} \cdot \exp \left(-\beta / T_{T A}\right) \\
& \beta=\frac{q}{k_{B} T_{\text {latt }}}, \quad \bar{a}_{\text {del }}=\frac{1.0}{N} \sum_{i=1}^{N} \frac{\partial \mid\left\langle k^{\prime}\left|H_{i}^{\prime}\right| k>\left.\right|^{3}\right.}{\partial T_{\text {latt }}} \cdot T_{\text {latt }}
\end{aligned}
$$

Where, $\mathrm{T}_{\text {latt }}$ is a lattice temperature, and $\mathrm{P}_{\mathrm{III}}$ is a valley-III occupation of electrons at given temperature. $\mathrm{T}_{\mathrm{TA}}$ is phonon energy of TA-mode $(220.6[\mathrm{~K}]) . H^{\prime}$ is a Hamiltonian of phonon scatterings, and $k$ is a wave number of carriers. The developed model well fits the Monte-Carlo simulation result.

\section{Mobility Model and Simulation Results}

We modeled the $\mu_{\mathrm{sr}}$ based on the developed physical model described in section 3 . The following equation shows the developed model.

$$
\begin{aligned}
& \mu_{s r}=\frac{1.0}{S\left(k^{\prime}\right)} \frac{1.0}{E_{e f f}^{2.0}} Z_{s c a t t} \cdot\left(\frac{T_{o p t}-T_{e}}{T_{o p t}}\right) \\
& =\frac{\mu_{s r 0}^{\prime} 0}{E_{e f f}^{2.0}}\left(1.0-\frac{T_{e}}{T_{\text {opt }}}\right)=\frac{\mu_{s r 0}^{\prime}}{E_{\text {eff }}^{2.0}}\left(1.0-\frac{\alpha \cdot T_{\text {latt }}^{2 / 3}}{T_{\text {opt }}\left(T_{\text {latt }}\right)}\right) \\
& \mu_{s r 0}^{\prime}=\frac{Z_{s c a t t}}{S\left(k^{\prime}\right)}, T_{o p t}=\left(1.0-P_{I I I}\right) T_{o p, a v}, T_{o p, a v}=\frac{551.4+686.1}{2.0}
\end{aligned}
$$

Where, $Z_{\text {scatt }}$ is the physical coefficient, and $\alpha$ is the model. SR spectrum density is, in general, determined by AFM measurement, so $\mathrm{S}\left(\mathrm{k}^{\prime}\right)$ is not a model parameter. Figure 2(a)(b) shows the comparison results of the simulated and experimental data about $\mu_{\mathrm{sr} 0}$ on (100) and on (110) surface,respectively.

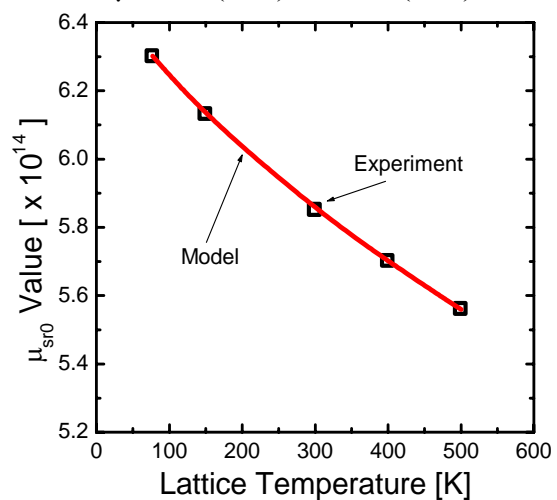

(a ) (100) Surface

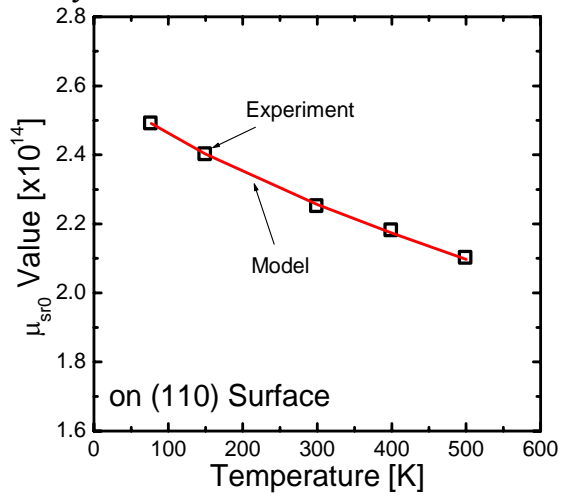

(b) (110) Surface

Figure 2 The comparison result of the simulated data to experimental data at given temperature on (100) surface. The developed model fit to the experimental data. 
Additionally, we show the mobility bahavior on (100) and (110) surface at 500K in Figure 3(a) and (b), respectively. The developed model can caputeure the experimental data although the surface orientation had changed.

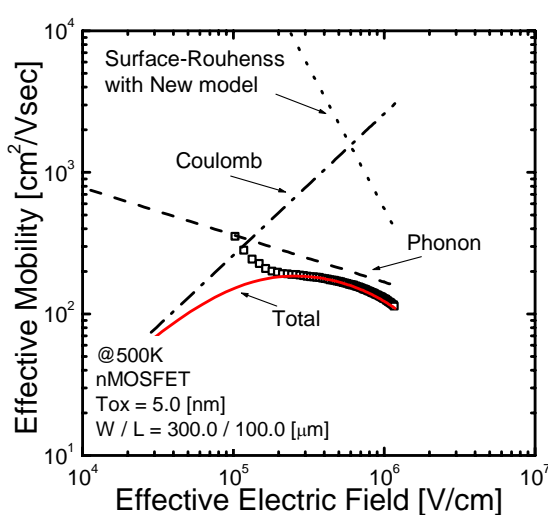

(a) (100) Surface

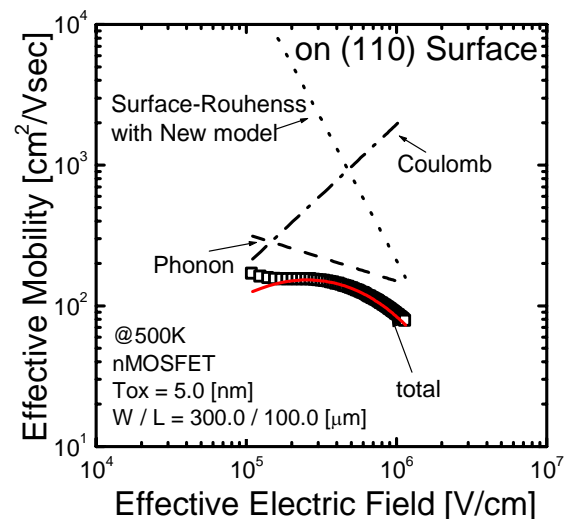

(b) (110) Surface

Figure 3 The comparison result on mobility behavior. The dots and line show the experimental data and simulated data using the developed model.

\section{Conclusion}

A compact mobility model of MOSFETs was developed based on the physics. The developed model can capture the mobility behavior at any temperature although the surface orientation has changed. This is because, based on the physics, the model equation is unified to any surface orientation. This model is key technology for designing SoC using the MOSFETs on any surface orientation.

\section{References}

[1] D. Su, M. Zargari, P. Yue, S. Rabii, D. Weber, B. Kaczynski, S. Mebta, K. Singh, S. Mendis, B. Wooley, "A 5GHz CMOS transceiver for IEEE 802.11a wireless LAN", ISSCC Dig. Tech., pp.70-74, 2002.

[2] P.T. M. van Zeijl, J-W. Eikenbroek ,P.-P. Vervoort, S. Setty, J. Tangenberg, G. Shipton, E. Kooistra, I. Keekstra, D. Belot, "A Bluetooth radio in $0.18 \mu \mathrm{m}$ CMOS", ISSCC Dig. Tech., pp.86-89, 2002.

[3] A. Teramoto, T. Hamada, T H. Akahori, K. Nii, T. Suwa, K. Kotani, M. Hirayama, M, S.Sugawa, T. Ohmi, "Low noise balanced-CMOS on $\mathrm{Si}(110)$ surface for analog/digital mixed signal circuits”, IEDM Tech. Dig., pp.801-803, 2003.

[4] S. Takagi, A. Toriumi, A. M. Iwase, H. Tango, "On the universality of inversion layer mobility in Si MOSFET's: Part I-effects of substrate impurity concentration", IEEE Trans, Vol. ED-41, pp. 2357 - 2362, 1994.

[5] HiSIM User's Guide.

[6] G. Mazzoni, A. Lacaita, L. M. Perron, A. Pirovano, "On surface roughness-limited mobility in highly doped n-MOSFET's", , IEEE Trans, Vol. ED-46, pp. 1423 - 1428, 1999. 